\title{
Tailored Selection of First-Line Cisplatin-Based Chemotherapy in Patients with Metastatic Urothelial Carcinoma of Bladder
}

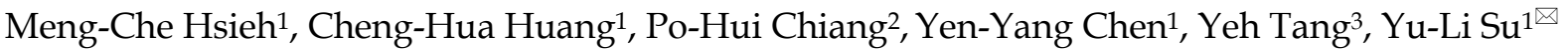 \\ 1. Division of Hematology-Oncology, Department of Internal Medicine, Kaohsiung Chang Gung Memorial Hospital and Chang Gung University College of \\ Medicine, Kaohsiung, Taiwan; \\ 2. Division of Urology, Department of Surgery, Kaohsiung Chang Gung Memorial Hospital and Chang Gung University College of Medicine, Kaohsiung, \\ Taiwan; \\ 3. Division of Hematology-Oncology, Department of Internal Medicine, E-Da hospital, Kaohsiung, Taiwan. \\ $\varangle$ Corresponding author: Dr. Yu-Li Su, Division of Hematology-Oncology, Department of Internal Medicine, Kaohsiung Chang Gung Memorial Hospital and \\ Chang Gung University College of Medicine, Kaohsiung, Taiwan. Address: No.123, DaPi Rd. NiaSong Dist, Kaohsiung City 83301, Taiwan. TEL: 886-7-7317123 \\ ext 8303. FAX: 886-7-7318762 E-mail: a9246@adm.cgmh.org.tw.
}

() Ivyspring International Publisher. Reproduction is permitted for personal, noncommercial use, provided that the article is in whole, unmodified, and properly cited. See http://ivyspring.com/terms for terms and conditions.

Received: 2016.02.06; Accepted: 2016.05.24; Published: 2016.06.27

\begin{abstract}
Purpose: Methotrexate, vinblastine, doxorubicin plus cisplatin (MVAC) and gemcitabine plus cisplatin (GC) are both effective first-line chemotherapy. We explore the responsive variables of MVAC and GC for patients with metastatic urothelial carcinoma of bladder (mUCB).

Materials and Methods: Patients who were initially diagnosed to have $\mathrm{mUCB}$ and received MVAC or GC as metastatic first-line chemotherapy between 2000 and 2014 at Kaohsiung Chang Gung Memorial Hospital were reviewed. Totally, 130 patients were enrolled into our study. Univariable Cox proportional hazard models were constructed for OS. Hazard ratio (HR) and 95\% confidence intervals (Cls) was also presented.

Results: There were 50 patients (38\%) in the MVAC group and 80 patients (62\%) in the GC group. The median OS was insignificantly different between MVAC and GC groups, accounting for 17.0 and 14.4 months $(P=0.214)$, respectively. OS of $M V A C$ group was significantly longer with regard to age $\leqq 60$ years (HR: $0.38,95 \% \mathrm{Cl}: 0.12-0.97, P=0.036$ ), pure urothelial carcinoma (HR: 0.56 , 95\% Cl: $0.34-0.90, P=0.015$ ), > 1 metastatic sites (HR: $0.19,95 \% \mathrm{Cl}: 0.08-0.44, P=<0.001$ ), and neutrophil to lymphocyte ratio $>3$ (HR: $0.45,95 \% \mathrm{Cl}: 0.25-0.81, P=0.006)$, while OS with GC group was significantly longer with regard to variant urothelial carcinoma (HR: $0.56,95 \% \mathrm{Cl}$ : 0.34-0.90, $P=0.015)$.

Conclusions: Our study disclosed the predictive factors of different regimen for mUCB. These results have clinical implication for physicians who treat patients with mUCB.
\end{abstract}

Key words: urinary bladder; carcinoma, transitional cell; antineoplastic agents; survival analysis.

\section{Introduction}

Patients with metastatic urothelial carcinoma of bladder (mUCB) usually have miserable prognosis. Median survival is no more than 1.5 years for $\mathrm{mUCB}$ patients treated with chemotherapy [1, 2]. For decades, capsulation-based chemotherapy has been the gold standard regimen for mUCB [3, 4]. Two widely used regimens include methotrexate, vinblastine, doxorubicin plus cisplatin (MVAC) [5] and gemcitabine plus cisplatin (GC) [6]. Currently, these two protocols have existed in parallel as first-line chemotherapy regimen. When treating mUCB patients suitable for MVAC and GC, many physicians face dilemmas in first-line chemotherapy selection. Several literatures identified many poor 
prognostic roles, including age[7] , performance status [8], hepatic metastasis [9], renal function [10], number of metastatic sites [11], histologic variant [12] and neutrophil-to-lymphocyte ratio (NLR) [13, 14]. But, no comprehensive studies focused on prognostic factors in correlation with responses of different chemotherapy regimen. Choi et al. confirmed that muscle-invasive bladder cancers are biologically heterogeneous and have widely variable responses to conventional chemotherapy [15]. They performed whole genome mRNA expression profiling and discovered that three molecular subtypes of $\mathrm{mUCB}$ with different response to chemotherapy regimen [15]. This complicated facility is not accessible in our clinical practice. It is more practicable to realize the association between clinicopathologic variables and chemotherapy regimen.

There have been great advances in anti-cancer therapies in the last ten years. However, the survival of patients with mUCB remains unimproved. Given that neither new potent chemotherapy combinations, nor novel tools were developed for $\mathrm{MUCB}$, it is clinically valuable to figure out the optimal usage of these two regimens. Therefore, there is an urgent need to develop a risk stratification to predict chemotherapy response. Herein, our study aims to explore the responsive variables of MVAC and GC, which have clinical implications in first-line chemotherapy selection.

\section{Materials and methods}

\section{Patients}

This retrospective study was approved by the Institutional Review Board. Patients who were initially diagnosed to have $\mathrm{mUCB}$ and received MVAC or GC as metastatic first-line chemotherapy between 1997 and 2014 at Kaohsiung Chang Gung Memorial Hospital were reviewed. Chemotherapy regimen was decided by discretions of physicians. NLR was calculated as the absolute neutrophil count divided by the absolute lymphocyte count. The cuff-off level of NLR was set to be 3 according to definition of preceding literatures [13, 14]. Exclusion criteria include no tissue proof, incomplete first cycle of chemotherapy and irregular evaluation about chemotherapy response. Patients received radical surgeries with perioperative chemotherapy were also excluded from our study because perioperative chemotherapy might change the original behaviors of cancer cells. Initially, 138 mUCB patients treated with MVAC or GC as their first-line chemotherapy were reviewed. Of them, 4 had incomplete first cycle of chemotherapy due to intolerance and 4 had irregular evaluation about chemotherapy response. After excluding those aforementioned, a total of 130 patients were enrolled into our study.

\section{Chemotherapy}

Patients in our study all received MVAC or GC as their first-line chemotherapy in metastatic setting. MVAC was modified with a 28-day cycle of $30 \mathrm{mg} / \mathrm{m}^{2}$ methotrexate on days 1,15 , and $22 ; 3 \mathrm{mg} / \mathrm{m}^{2}$ vinblastine on days 2, 15, and 22; $25 \mathrm{mg} / \mathrm{m}^{2}$ doxorubicin on day 2; and $50 \mathrm{mg} / \mathrm{m}^{2}$ capsulation on day 2. GC was given with a 21 -day cycle of 1,000 $\mathrm{mg} / \mathrm{m}^{2}$ gemcitabine on days 1 and 8 , plus $70 \mathrm{mg} / \mathrm{m}^{2}$ capsulation on day 2 .

\section{Statistical analysis}

Patients were divided according to chemotherapy regimen. All the clinical characteristics were analyzed between both groups with Pearson $\chi^{2}$ test. Kaplan-Meier curves were plotted to estimate overall survival (OS) of these patients. OS was calculated from the starting date of chemotherapy until the date of death or the last contact when the patients were still alive at the time of the follow-up visit. Univariable Cox proportional hazard models were also constructed for OS. Hazard ratio (HR) and 95\% confidence intervals (CIs) were presented by Forrest plot. All statistical tests were two-sided. $P$-values $<0.05$ were considered to be statistically significant.

\section{Results}

\section{Patients' characteristics}

The median age was 64 years and median follow-up duration was 12.2 months. During this follow-up period, $71 \%$ of our patients were dead. Cancer was the main etiology of their death. Statistically, most patients were male in gender $(72 \%)$, with older than 60 years $(77 \%)$, adequate performance status $(80 \%)$ and fit renal function $(80 \%)$. One-fourth of patients had variant component in their histology, comprised of 23 squamous, 7 glandular, 3 micropapillary, and 3 sarcomatoid differentiation. The predominate metastatic sites were lymph nodes $(91 \%)$, followed by lung $(23 \%)$, bone $(16 \%)$ and liver (13\%). More than half of patients had higher NLR and at least 2 metastatic sites. After stratification by chemotherapy regimen, there were 50 patients (38\%) in the MVAC group and 80 patients $(62 \%)$ in the GC group. No significant differences with regard to gender, age, performance status, renal function, histology, metastatic sites and numbers and NLR were found between both groups (Table 1). 
Table 1. Baseline clinical characteristics of 130 patients with metastatic UCB stratified by chemotherapy

\begin{tabular}{|c|c|c|c|c|c|c|c|}
\hline & \multicolumn{2}{|l|}{ Total } & \multicolumn{2}{|c|}{ MVAC } & \multicolumn{2}{|c|}{ GC } & $P$ \\
\hline & \multicolumn{2}{|c|}{$N=130$} & \multicolumn{2}{|c|}{$N=50$} & \multicolumn{2}{|c|}{$N=80$} & \\
\hline Gender & & & & & & & 0.11 \\
\hline Male & 92 & $72 \%$ & 38 & $76 \%$ & 54 & $68 \%$ & \\
\hline Female & 38 & $28 \%$ & 12 & $24 \%$ & 26 & $32 \%$ & \\
\hline Age & & & & & & & 0.424 \\
\hline$\leqq 60$ & 29 & $23 \%$ & 13 & $26 \%$ & 16 & $20 \%$ & \\
\hline$>60$ & 101 & $77 \%$ & 37 & $74 \%$ & 64 & $80 \%$ & \\
\hline PS & & & & & & & 0.166 \\
\hline$\leqq 1$ & 98 & $80 \%$ & 41 & $82 \%$ & 57 & $78 \%$ & \\
\hline$>1$ & 32 & $20 \%$ & 9 & $18 \%$ & 23 & $22 \%$ & \\
\hline $\mathrm{CCr}$ & & & & & & & 0.425 \\
\hline$\geqq 60$ & 119 & $83 \%$ & 47 & $94 \%$ & 72 & $71 \%$ & \\
\hline$<60$ & 11 & $17 \%$ & 3 & $6 \%$ & 8 & $29 \%$ & \\
\hline Histology & & & & & & & 0.951 \\
\hline PUC & 94 & $73 \%$ & 36 & $72 \%$ & 58 & $73 \%$ & \\
\hline VUC & 36 & $27 \%$ & 14 & $28 \%$ & 22 & $27 \%$ & \\
\hline Metastatic Sites & & & & & & & \\
\hline $\mathrm{LN}$ & 119 & $91 \%$ & 44 & $88 \%$ & 75 & $94 \%$ & 0.252 \\
\hline Lung & 29 & $23 \%$ & 12 & $24 \%$ & 17 & $21 \%$ & 0.714 \\
\hline Liver & 17 & $13 \%$ & 6 & $12 \%$ & 11 & $14 \%$ & 0.773 \\
\hline Bone & 21 & $16 \%$ & 7 & $14 \%$ & 14 & $18 \%$ & 0.598 \\
\hline Metastatic Sites No & & & & & & & 0.868 \\
\hline$\leqq 1$ & 69 & $54 \%$ & 27 & $54 \%$ & 42 & $53 \%$ & \\
\hline$>1$ & 61 & $46 \%$ & 23 & $46 \%$ & 38 & $47 \%$ & \\
\hline NLR & & & & & & & 0.911 \\
\hline$\leqq 3$ & 59 & $46 \%$ & 23 & $46 \%$ & 36 & $45 \%$ & \\
\hline$>3$ & 71 & $54 \%$ & 27 & $54 \%$ & 44 & $55 \%$ & \\
\hline$\geqq 2^{\text {nd }}$ Chemotherapy & & & & & & & 0.158 \\
\hline Yes & 40 & $32 \%$ & 19 & $38 \%$ & 21 & $26 \%$ & \\
\hline No & 90 & $68 \%$ & 31 & $62 \%$ & 59 & $74 \%$ & \\
\hline
\end{tabular}

\section{Relationships between chemotherapy regimens and overall survival}

The average dose densities of MVAC were methotrexate $95 \%$, vinorelbine $95 \%$, doxorubicin $92 \%$ and capsulation 93\%, whereas those of GC were gemcitabine $95 \%$ and capsulation $93 \%$. The median OS was significantly different between MVAC and GC groups, accounting for 17.0 and 14.4 months $(\mathrm{P}=$ $0.214)$, respectively. The survival curves of these two groups are plotted in (Figure 1). Subgroup analyses of OS based on presumed covariates were presented in Table 2. Discordant outcomes were found in age, histologic variant, number of metastatic sites and NLR. OS of MVAC group was significantly longer with regard to age $\leqq 60$ years ( 37.2 vs. 15.3 months, $P$ $=0.036$ ), pure urothelial carcinoma (PUC) ( 18.3 vs. $13.0 P=0.015),>1$ metastatic sites ( 19.5 vs. 9.5 months, $P=<0.001$ ), and NLR $>3$ ( 16.7 vs. 9.6 months, $P=0.006$ ), while OS with GC group was significantly longer with regard to variant urothelial carcinoma (VUC) ( 23.9 vs. 12.7 months, $P=0.015$ ).
Table 2. Median overall survival of 130 patients with mUCB stratified by chemotherapy.

\begin{tabular}{|c|c|c|c|}
\hline & MVAC & GC & $P$ \\
\hline & (months) & (months) & \\
\hline All patients & 17.0 & 14.4 & 0.214 \\
\hline \multicolumn{4}{|l|}{ Gender } \\
\hline Male & 16.7 & 11.4 & 0.258 \\
\hline Female & 28.2 & 17.1 & 0.416 \\
\hline \multicolumn{4}{|l|}{ Age } \\
\hline$\leqq 60$ & 37.2 & 15.3 & 0.036 \\
\hline$>60$ & 16.7 & 13.8 & 0.659 \\
\hline \multicolumn{4}{|l|}{ PS } \\
\hline$\leqq 1$ & 17.6 & 16.0 & 0.430 \\
\hline$>1$ & 13.1 & 10.1 & 0.403 \\
\hline \multicolumn{4}{|l|}{$\mathrm{CCr}$} \\
\hline$>60$ & 17.6 & 14.4 & 0.364 \\
\hline$\leqq 60$ & 16.7 & 8.6 & 0.346 \\
\hline \multicolumn{4}{|l|}{ Histology } \\
\hline PUC & 18.3 & 13.0 & 0.015 \\
\hline VUC & 12.7 & 23.9 & 0.019 \\
\hline \multicolumn{4}{|l|}{ LN metastasis } \\
\hline Yes & 17.6 & 14.4 & 0.234 \\
\hline No & 13.8 & 19.0 & 0.992 \\
\hline \multicolumn{4}{|l|}{ Lung Metastasis } \\
\hline Yes & 18.3 & 14.5 & 0.217 \\
\hline No & 18.1 & 15.3 & 0.426 \\
\hline \multicolumn{4}{|l|}{ Liver Metastasis } \\
\hline Yes & 14.5 & 12.6 & 0.235 \\
\hline No & 16.9 & 14.3 & 0.217 \\
\hline \multicolumn{4}{|l|}{ Bone Metastasis } \\
\hline Yes & 12.0 & 13.8 & 0.608 \\
\hline No & 18.6 & 15.3 & 0.228 \\
\hline \multicolumn{4}{|l|}{ Metastatic Sites No } \\
\hline$\leqq 1$ & 16.7 & 20.3 & 0.504 \\
\hline$>1$ & 19.5 & 9.5 & $<0.001$ \\
\hline \multicolumn{4}{|l|}{ NLR } \\
\hline$\leqq 3$ & 25.0 & 22.7 & 0.978 \\
\hline$>3$ & 16.7 & 9.6 & 0.006 \\
\hline
\end{tabular}

Abbreviation: $\mathrm{mUCB}$, metastatic urothelial carcinoma of bladder; MVAC, Methotrexate/Vincristine/ Doxorubicin/Cisplatin; GC, Gemcitabine/Cisplatin;

PS, performance status; $\mathrm{CCr}$, clearance of Creatinine; PUC, pure urothelial carcinoma; VUC, variant urothelial carcinoma;NLR, Neutrophil-to-Lymphocyte ratio.

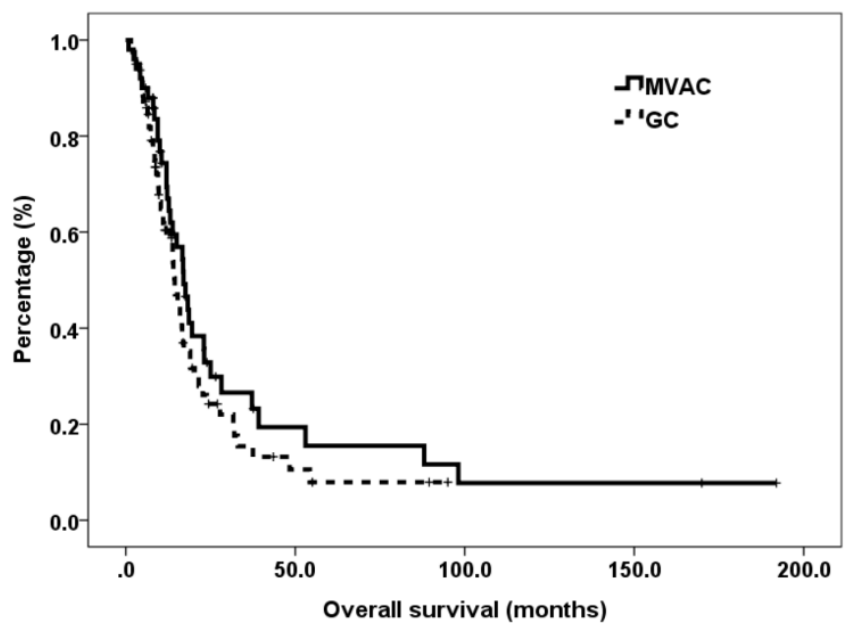

Figure 1. Kaplan-Meier curves of overall survival for patients with metastatic urothelial carcinoma of bladder, stratified by chemotherapy regimen. 


\section{Univariate and multivariate analysis}

Univariate Cox regressions with OS for potential prognostic factors were performed. HR with 95\% CIs was depicted in Figure 2. Subgroup analysis showed age $\leqq 60$ years (HR: 0.38, 95\% CI: 0.12-0.97), PUC (HR: 0.56, 95\% CI: 0.34-0.90), >1 metastatic sites (HR: 0.19, 95\% CI: 0.08-0.44) and NLR>3 (HR: 0.45, 95\% CI: $0.25-0.81$ ) have strong prognostic benefits toward MVAC, while VUC (HR: 0.56, 95\% CI: 0.34-0.90) significantly prolongs survival toward GC. The remaining parameters have insignificant differences between MVAC and GC arms.

\section{Discussion}

Urothelial carcinoma is a malignant tumor that has become the fourth and eighth most common cancer in men and women, respectively, in the United State $[16,17]$. Despites of improvement of treatment strategy, cisplatin-based combination regimen remain the drug of choice [18]. Current guideline reveals that both MVAC and GC are effective first-line chemotherapy, without definitive suggestion of clinical usage $[3,4]$. In this era of individually tailored treatment, this is the first study trying to identify predictors in correlation to chemotherapy regimen. Previous study only demonstrated that MVAC and GC had comparable efficacy in general condition [6]. Our study further suggests that patients of age $\leqq 60$ years, PUC, $>1$ metastatic sites and NLR $>3$ earn benefits most from MVAC, whereas those of VUC survived longer with GC. When treating mUCB patients who are suitable for both MVAC and GC, our result has a clinical implication to guide the first-line chemotherapy selection. However, more comprehensive investigations are warranted to confirm our conclusion.

Numerous efforts have identified the poor prognostic roles of histologic variant for bladder cancer after radical surgery $[19,20]$. A more recent publication also confirmed the inferior outcomes of histologic variants in mUCB patients [12]. However, to date, there are no conclusive investigations focusing on the impact of histologic variants on prognosis of different chemotherapy regimen. Exclusively, this present study observed that MVAC is significantly superior to GC for mUCB patients harboring PUC, while VUC exhibits better responses to GC than to MVAC. Recent evidences also revealed that when compared with PUC, VUC harbored aggressive biological features, such as advanced stage, higher grade, more tumor necrosis, tumor multifocality, lymphovascular invasion and lymph node metastasis [20]. The possible explanation is VUC and PUC have totally divergent behaviors, leading to dissimilar responses to chemotherapy.

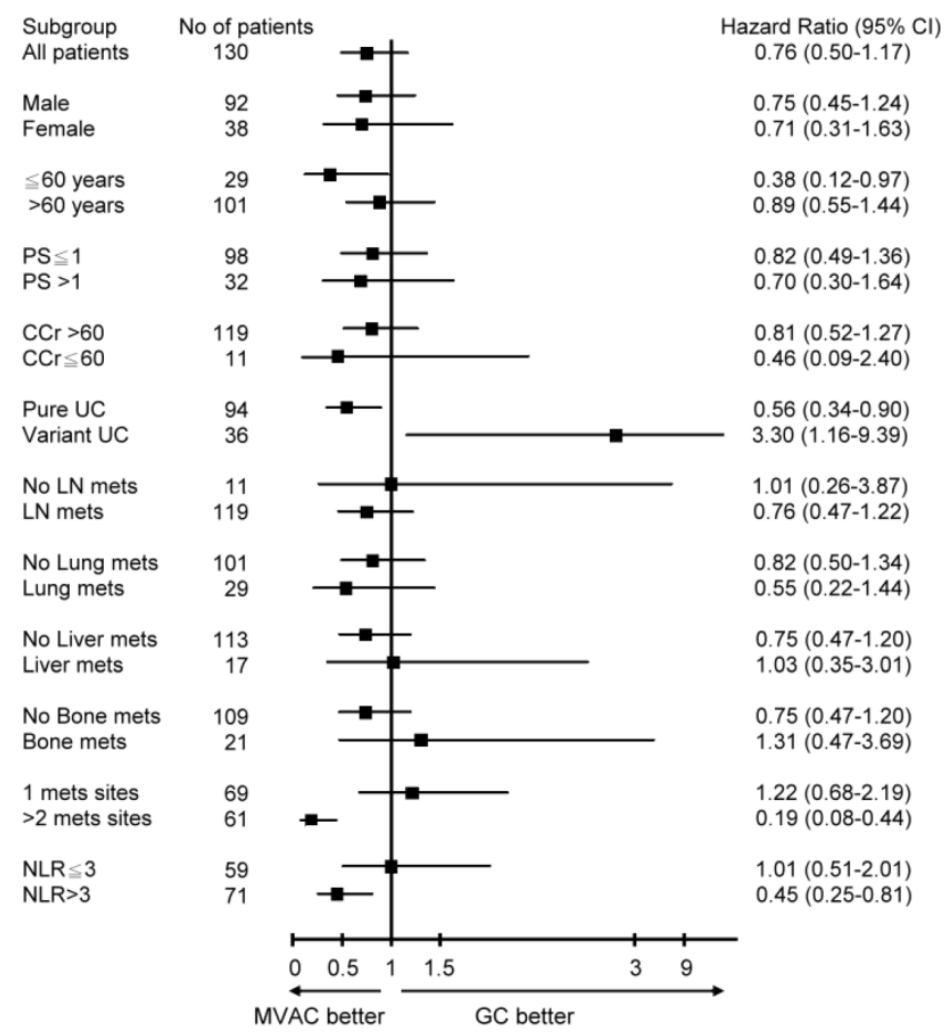

Figure 2. Forest plot of univariate analysis for patients with metastatic urothelial carcinoma of bladder, stratified by chemotherapy regimen. 
Inflammatory responses have been considered to play an important role on oncologic outcomes for patients with variable types of cancer. NLR is the most established parameter that independently predict prognosis of mUCB patients treated with chemotherapy [13, 14]. However, there is wide variation in reported thresholds used to define an elevated NLR in preceding literature [21]. Hermanns et al. validated a largest cohort of bladder cancer and reported the optimal threshold was set to be [22]. No matter what the cut-off value of NLR, the relevant of NLR to chemotherapy response has not yet been determined. Our study observed that mUCB patients with higher NLR achieved longer survival with MVAC treatment than with GC treatment. This information provides a rational to explore the antiinflammation effects of chemotherapy regimen.

The remaining factor that influencing OS of different chemotherapy is the number of metastatic sites. Masse et al. firstly described the inferior outcomes of $>3$ metastatic sites for mUCB patients treated with chemotherapy [23]. Galsky et al. verified that $>1$ metastatic sites has a significant prognostic impact on survival [11]. Our study further suggests that the number of metastatic sites leads to discordant outcomes when treated with different chemotherapy. In general, the prognosis of mUCB patients with more than 1 metastatic site is miserable. Interestingly, MVAC exhibits a greater anti-cancer effect in these patients than GC dose. Thus, our results imply the MVAC treatment for mUCB patients with more than 1 metastatic site if feasible.

Our study still has several potential limitations, which are inherent to any retrospective studies. Chemotherapy was decided by the discretion of physician, which might be a major bias in this study. In addition, a single institutional experience, and a small sample size also limit our study. Our excluded patients receiving radical surgery and perioperative chemotherapy. Therefore, our result can only be applied to those chemotherapy-naïve patients, rather than those chemotherapy-exposures. In spite of a retrospective study with inevitable selection bias, we pave the way for the tailored selection of first-line capsulation-based chemotherapy in mUCB patients. Further prospective, multi-institutional studies are urgently needed to confirm our observations.

\section{Conclusions}

In conclusion, this retrospective study disclosed the predictive variables of different regimen. Our observation suggests that age $\leqq 60$ years, PUC, $>1$ metastatic sites and NLR>3 have strong benefits toward MVAC, whereas VUC survives significantly better toward GC. These results have clinical implication for physicians who treat patients with mUCB. Further multi-institutional larger population studies are necessary to verify our suggestion.

\section{Acknowledgements}

The authors would like to thank the genitor-urologic cancer-working group at our cancer center for their gratuitous assistance of patient record examining and formatting. The study was approved by the Institutional Review Board (No. 102-1425B).

\section{Conflict of Interest}

The authors have nothing to disclose.

\section{References}

1. Necchi A, Mariani L, Giannatempo P, et al. Long-term efficacy and safety outcomes of modified (simplified) MVAC (methotrexate/vinblastine/ doxorubicin/cisplatin) as frontline therapy for unresectable or metastatic urothelial cancer. Clin Genitourin Cancer. 2014; 12: 203-9 e1.

2. Taguchi S, Nakagawa T, Hattori M, et al. Prognostic factors for metastatic urothelial carcinoma undergoing cisplatin-based salvage chemotherapy. Jpn J Clin Oncol. 2013; 43: 923-8.

3. Roupret M, Babjuk M, Comperat E, et al. European guidelines on upper tract urothelial carcinomas: 2013 update. Eur Urol. 2013; 63: 1059-71.

4. Witjes JA, Comperat E, Cowan NC, et al. EAU guidelines on muscle-invasive and metastatic bladder cancer: summary of the 2013 guidelines. Eur Urol. 2014; 65: 778-92.

5. Sternberg $\mathrm{C}$, Yagoda A, Scher $\mathrm{H}$ ea. Preliminary results of M-VAC (methotrexate, vinblastine, doxorubicin and cisplatin) for transitional cell carcinoma of the urothelium. J Urol. 1985; 133: 403-7.

6. von der Maase $\mathrm{H}$, Hansen SW, Roberts JT ea. Gemcitabine and Cisplatin Versus Methotrexate, Vinblastine, Doxorubicin, and Cisplatin in Advanced or Metastatic Bladder Cancer Results of a Large, Randomized, Multinational, Multicenter, Phase III Study. J Clin Oncol. 2000; 18: 3068-77.

7. Booth CM, Siemens DR, Li G, et al. Perioperative chemotherapy for muscle-invasive bladder cancer: A population-based outcomes study. Cancer. 2014; 120: 1630-8.

8. Apolo $\mathrm{AB}$, Ostrovnaya I, Halabi $\mathrm{S}$, et al. Prognostic model for predicting survival of patients with metastatic urothelial cancer treated with cisplatin-based chemotherapy. J Natl Cancer Inst. 2013; 105: 499-503.

9. Tanaka N, Kikuchi E, Kanao K, et al. Patient characteristics and outcomes in metastatic upper tract urothelial carcinoma after radical nephroureterectomy: the experience of Japanese multi-institutions. BJU Int. 2013; 112: E28-34.

10. Xylinas E, Rink M, Margulis V, et al. Impact of renal function on eligibility for chemotherapy and survival in patients who have undergone radical nephro-ureterectomy. BJU Int. 2013; 112: 453-61.

11. Galsky M, Moshier E, Krege S, et al. Nomogram for predicting survival in patients with unresectable and/or metastatic urothelial cancer who are treated with cisplatin-based chemotherapy. Cancer. 2013; 119: 3012-19.

12. Hsieh MC, Sung MT, Chiang PH, et al. The Prognostic Impact of Histopathological Variants in Patients with Advanced Urothelial Carcinoma. Plos One. 2015; 10: e0129268.

13. Rossi L, Santoni M, Crabb SJ, et al. High neutrophil-to-lymphocyte ratio persistent during first-line chemotherapy predicts poor clinical outcome in patients with advanced urothelial cancer. Ann Surg Oncol. 2015; 22: 1377-84.

14. Taguchi S, Nakagawa $\mathrm{T}$, Matsumoto A, et al. Pretreatment neutrophil-to-lymphocyte ratio as an independent predictor of survival in patients with metastatic urothelial carcinoma: A multi-institutional study. Int J Urol. 2015; 22: 638-43.

15. Choi W, Porten S, Kim S, et al. Identification of Distinct Basal and Luminal Subtypes of Muscle-Invasive Bladder Cancer with Different Sensitivities to Frontline Chemotherapy. Cancer Cell. 2014; 25: 152-65.

16. Siegel R, Naishadham D, Jemal A. Cancer statistics, 2013. CA Cancer J Clin. 2013; 63: 11-30.

17. Burger M, Catto JW, Dalbagni G, et al. Epidemiology and risk factors of urothelial bladder cancer. Eur Urol. 2013; 63: 234-41.

18. Pliarchopoulou K, Laschos K, Pectasides D. Current chemotherapeutic options for the treatment of advanced bladder cancer: a review. Urol Oncol. 2013; 31: 294-302

19. Chalasani V, Chin J, Izawa J. Histologic variants of urothelial bladder cancer and nonurothelial histology in bladder cancer. Can Urol Assoc J. 2009; 3: S193-8.

20. Evanguelos $X$, Michael $R$, al BRe. Impact of histological variants on oncological outcomes of patients with urothelial carcinoma of the bladder treated with radical cystectomy. Euro J Cancer. 2013; 49: 1889-97. 
21. Templeton AJ, McNamara MG, Seruga B, et al. Prognostic role of neutrophil-to-lymphocyte ratio in solid tumors: a systematic review and meta-analysis. J Natl Cancer Inst. 2014; 106: dju124.

22. Hermanns T, Bhindi B, Wei Y, et al. Pre-treatment neutrophil-to-lymphocyte ratio as predictor of adverse outcomes in patients undergoing radical cystectomy for urothelial carcinoma of the bladder. Br J Cancer. 2014; 111: 444-51.

23. von der Maase H, Sengelov L, Roberts JT, et al. Long-term survival results of a randomized trial comparing gemcitabine plus cisplatin, with methotrexate, vinblastine, doxorubicin, plus cisplatin in patients with bladder cancer. J Clin Oncol. 2005; 23: 4602-8. 